\title{
Subject Index Vol. 191,1995
}

Acid mantle 276

Acne models 305

Action spectrum 286

Acute lymphocytic leukemia 333

Adjuvant immunotherapy 234

Alopecia 273

- areata 68

Alpha 1-antitrypsin 204

Amino levulinic acid 346

Amyloid 56

Amyloidosis, cutaneous 56

Anetoderma 204

Angiocentric T-cell lymphoma 336

Angiosarcoma 357

-, head and neck 357

Antibody 119

Antifungal activity 311

Antihistone antibodies 16

Anti-melanoma monoclonal antibody 119

Antiphospholipid (aPL) antibodies 204

Asplenia 68

Atopic dermatitis 72

Augmentation spectrum 286

Autoimmune disease 39

Bacterial flora 276

Basic fibroblast growth factor 281

Bilateral distribution 157,260

Biomechanics 9

Bloch-Sulzberger syndrome 163

Bony resorption 62

Borrelia afzelii 193

burgdorferi sensu stricto 193

garinii 193 Bullous disease 39 Burn scar 328 Bursitis, multiple 318

Calcipotriol, topical 341

Calcitriol 226

Candidiasis-endocrinopathy syndrome 68

Capsaicin 73

CD-34 154

Cell proliferation 226 
Cheilitis granulomatosa 252

Chemotherapy 65

CHILD nevus 210

- $\quad$ syndrome 210

Childhood 264

Chronic myeloid leukemia 321 Classification 104 Coal tar gel 311 Colibiogene ${ }^{\circledR} 343$

Collagen 115

disease 318

synthesis 226 Collar 82

Combined surgical instrumentation 240 Condylomata acuminata 129 Congenital hemidysplasia

210 Contact allergy 109 Cornified cell envelope 124 Corticosteroids 109 Cost 199

- reduction 240

Cross-reactivity 109

Cutis verticis gyrata 292

Cycloserine 142

Cystic lesion 318

Cytokines 273

Cytomegalovirus 159,269

Dandruff 311 Dermal eruptions 240

- $\quad$ fíbroblasts 226

Dermatitis herpetiformis 88, 168

Dermatomyositis 242

Dermis 9 Diclofenac 133 Differential diagnosis 350 Diphenylcyclopropenone 68 Drug rash 76

Elasticity 9

Elastolysis 19

Eosinophilia 133

Eosinophilic pustular folliculitis 257

Epidermal differentiation 124

nevus 210 Epithelial hood 82

skirt 82

Erythropoietic protoporphyria 36 Evolution 199

Extracorporeal photochemotherapy 25 Eyelids 350

F(ab')2 119 Fat lobules 149 Fibrillar deposition 88 Fibroblast growth factor 281 Fibroelastolytic patterns 19 Fixed drug eruption 185,270 Fluoxetine 166 Follicular accentuation 268

- $\quad$ hyperkeratosis 242

Free radicals 36

Functional X-chromosome mosaicism 1 Fusarium 333

Gametic half-chromatid mutation 1 Gamma-interferon 99 Genetic mosaicism 1 Genital herpes

32,72

- $\quad$ warts 129

Gianotti-Crosti syndrome 340 Gloves-and-socks syndrome 269 Gluten-sensitive enteropathy 88 Glycosaminoglycan 115

Haemangioma 315 Hair, human 273 Hamartoma 1 Helicobacter pylori 6 Hemostasis 240

Hepatitis C 43 Hereditary hypotrichosis 139 Herpes simplex virus 159 
- $\quad$ - - type 272

HIV 56,159,259,348

HLA 88

Hobnail hemangioma 154 Human papillomavirus 129 Hydroxyethylrutosides 36 Hydroxyurea

321 Hyperpigmentation 65, 163 Hypertrichosis lanuginosa acquisita 82 Hypohidrotic ectodermal dysplasia 355

Ichthyosiform nevus 210

Ichthyosis 323

IgA deficiency 46

ILVEN 210

Imaging 93

Immunofluorescence 88

Immunoglobulins 46

Immunology 46

Immunophenotyping 234

Immunoprecipitation 39

In vitro susceptibility 311

Incontinenta pigmenti 163

Inflammation 273

Inflammatory linear verrucous epidermal

nevus 210 Influenza virus vaccination 340 Inhibition spectrum 286 Interferon alfa-2b 234,315

Interferon-alpha 43,257

- $\quad$ (leucocyte) 129

Interleukin 1273

Intraepidermal IgA pustulosis 352

Involution 149

Kaposi 188

Kaposi's sarcoma (retrospective study) 104

Keratosis lichenoides chronica 188,264

LI7M regimen 336 Leiomyomas cutaneous 295 Lethal midline granuloma 336 Leucocyte

interferon-alpha 32,129 Leukocytoclastic vasculitis 268 Lichen planus 72, 350

- ruber acuminatus verrucosus et reticu-

laris 188

Lichenoid drug eruption 142 Light 93

- reaction, persistent 343

Limb defects 210

Lip swelling 252 Lipoatrophy 149 Lung carcinoma 59 Lupus (SLE) 204,281 Lyme disease 193

Macrophage 149

- $\quad$ colony stimulating factor 315

Malassezia furfur 311

-, quantification 348

Malignant hemangioendothelioma 357

Mantle hair 82

Mast cell 281

Mechanical properties 9

Melanoma 119,199,234, 328 
-, stage III B 234

Melkersson-Rosenthal syndrome 252

Mental retardation 292

Meralgia paraesthetica 73

Metastases 119,199

Metastatic melanoma drug therapy 234

Metronidazole 6,252

Mibelli 62

Microscopy 93

Microthrombosis 204

Milia en plaque $157,260,262$

Milium 249

Minimum inhibitory concentration 311

Mixed cryoglobulinemia 43

Molluscum contagiosum 167

Morphea 16,25

Mucositis 52

Multiple dermatofibromas 281

Mutilations 62

Nail pigmentation 342

Necrotizing vasculitis 43

Neoplasias 240

NERDS syndrome 81,133

Neuralgia 76

Nevoid disorders 1

Nevus 1

Nodules, para-articular 133

Nude mice 99

Oat cell 59 Ofuji's disease 257 Olaquindox 343

369

Ondansetron 270

Optical coherence microscopy 93

Otitis 355

Pain 295

Panniculitis 49

Papillomatosis, confluent 341

-, reticulated 341

PAPS 204

Paraneoplastic pemphigus 39

Patch test reproducibility 109

testing 109 ü-Penicillamine 25 Perianal eczema 299

region 249

Periarticular rheumatism $318 \mathrm{pH} 276$

Pharmaceutical creams 32,129 Photoaging 124 Photosensitivity 286 Phototesting 286 Plasma cell 52 Plasmoacanthoma 52 Pleomorphic fibroma 245 Podophyllotoxin 129 Polymorphic reticulosis 336 Porokeratosis 62 Preservative 299 Protein tyrosine kinase 217 Provoking drugs 
217 Pseudoxanthoma elasticum 19 Psoriasis 46,72,217,254 Psychiatric population 292 PUVA 254

Recombinant human granulocyte 315

Recycling 299

Reticular erythematous mucinosis 159

Retinoicacid 124

Retinoid 305

Rheumatism 133

Rosacea 6

Rosai-Dorfman disease 49

Roxithromycin 342

Sarcoid granulomas 59 Scalp 245,321

- $\quad$ hair 139

Scintigraphy 119

Scleroderma 16,115,226

Sebaceous gland 82,305,355

Seborrhoeic dermatitis 311

Sebum 305 Senile lip licking 339 Sensitization 68 Sinus histiocytosis 49 Skin aging 19,99

biopsy price 240

cleansing 276

grafts 99

Sneddon-Wilkinson disease 352 Solar urticaria 286

Somatic mutation 1 Squamous-cell carcinoma 321,328 Stem cell factor 281 Stimulating factor (rhGM-CSF) 315 Summer improvement 264 Supraclavicular area 262 Supravenous skin 65

Surveillance 199 Survivorship function 104 Syndet 276 Syringoma 249 Systemic scleroderma 74

- $\quad$ sclerosis 25

T cells 217

T-cell lymphoma 352

Targetoid hemosiderotic hemangioma 154

Toilet paper 299

Topical photodynamic therapy 346

Tuberculosis 145

-, cutaneous 145

- $\quad$ verrucosa cutis 145

Tubular bones, symmetrical shortening 323 Tumor-related dermatosis 59 Tumors, cutaneous 295

Ulcer healing 315 Urtical vasculitis 166 Urticaria 133 Uterine myoma 295

Venereal disease 32,129

- $\quad$ warts 129

Verrucae vulgares 346

Verruciform xanthoma 210,254

White fibrous papulosis 19

X-linked dominant chondrodysplasia punctata 323

- $\quad$-, male-lethal inheritance 210

Xerosis 133 
Subject Index Vol. 191,1995 